\title{
Efeitos da Exposição a Instruções Correspondentes e Discrepantes sobre o Comportamento de Seguir Regras em Crianças em Idade Escolar*
}

\section{Effects of Exposure to Corresponding and Discrepant Instructions on the Behavior of Following Rules for School Age Children}

\section{Efectos de la Exposición a Instrucciones Correspondientes y Discrepantes sobre el Comportamiento de Seguir Reglas de Niños en Edad Escolar}

\author{
Taís da Costa Calheiros ${ }^{1}$, Luís Antônio Lovo Martins², Ana Paula Ollier e Silva ${ }^{3}$, Polyana Carla \\ Magon $^{4}$, Marcos Roberto Garcia ${ }^{5}$ e João Juliani ${ }^{6}$
}

[1] Primeiro autor. Centro Universitário Filadélfia, Londrina, Paraná, Brasil [2] Universidade de São Paulo [3] [4] [5] [6] Centro Universitário Filadélfia I Título abreviado: Confiabilidade e Seguimento de Regra I Endereço para correspondência: Rua Paranaguá, 1057 apto 204 cep: $86020-030$ - Londrina - Paraná Brasil | Email: calheirostais@gmail.com

Resumo: O seguimento de regra pode ser influenciado pela confiabilidade no falante? $\mathrm{O}$ objetivo desta pesquisa consistiu em verificar os efeitos de instruções correspondentes/discrepantes sobre o comportamento de seguir regras em condições com reforço imediato e na ausência de reforçamento diferencial. Dezesseis crianças de 5 a 9 anos participaram do estudo, sendo oito em ambos os experimentos. Em cada experimento, elas foram alocadas em dois grupos. A tarefa da criança era escolher entre cores, seguindo ou não a instrução apresentada pelo fantoche. Os experimentos foram divididos em fases (ABC Experimento I e ABCD Experimento II) e cada uma continha duas condições (Treino - reforçamento diferencial das respostas de escolha e Teste - sem reforçamento diferencial). As fases se diferenciavam em função da porcentagem de instruções correspondentes (Fase A - 100\%; B - 50\%; C - 0\%; $\mathrm{D}$ - repetição da primeira fase). Os grupos que iniciaram com a Fase A apresentaram maior consistência no seguimento de regra $(69,29 \%)$ comparados com os grupos que iniciaram com a Fase C $(61,96 \%)$. Esses resultados mostram que a confiabilidade no falante está relacionada com o fato da regra ser correspondente às consequências produzidas pelo seu seguimento.

Palavras-chave: comportamento governado por regras, controle instrucional, comportamento verbal, confiabilidade.

\begin{abstract}
Can rule-following be affected by the reliability of the speaker? The objective of this research was to determine the effects of corresponding/discrepant instructions on the behavior of following rules with immediate reinforcement conditions and in the absence of differential reinforcement. Sixteen children between the ages of 5 and 9 participated in the study, eight in both experiments. In each experiment, they were divided into two groups. The child's task was to choose between colors, following or not the instruction presented by a puppet. The experiments were divided into phases (ABC Experiment I and ABCD Experiment II) and each one contained two conditions (Training - differential reinforcement of choice answers and Test without differential reinforcement). The phases were distinguished in function to the percentage
\end{abstract}


of corresponding instructions (Phase A - 100\%; B - 50\%; C - 0\%; D - repeating the first phase). The groups that began with the Phase A presented a higher consistency on the rule-following $(69,29 \%)$ compared with the groups that started with the Phase C (61,96\%). Those results show that the reliability in the speaker is related to the fact that the rule can correspond with the consequences produced by its following.

Keywords: rule-governed behavior, instructional control, verbal behavior, reliability.

Resumen: ¿El seguimiento de regla puede ser influenciado por la confiabilidad en el hablante? El objetivo de esta investigación consistió en verificar los efectos de instrucciones correspondientes/discrepantes sobre el comportamiento de seguir reglas en condiciones con refuerzo inmediato y en la ausencia de refuerzo diferencial. Dieciséis niños de 5 a 9 años participaron del estudio, siendo ocho en ambos experimentos. En cada experimento, ellos fueron divididos en dos grupos. La tarea del niño era escoger entre colores, siguiendo o no la instrucción presentada por el títere. Los experimentos fueron divididos en fases (ABC Experimento I y ABCD Experimento II) y en cada una había dos condiciones (Entrenamiento - refuerzo diferencial de las respuestas de elección y Test - sin refuerzo diferencial). Las fases se diferenciaban en función del porcentaje de instrucciones correspondientes (Fase A - el $100 \%$; B - el 50\%; C - 0\%; D - repetición de la primera fase). Los grupos que iniciaron con la Fase A presentaron mayor consistencia en el seguimiento de regla (el 69,29\%) comparados con los grupos que iniciaron con la Fase C (el 61,96\%). Esos resultados muestran que la confiabilidad en el hablante está relacionada con el hecho de la regla ser correspondiente a las consecuencias producidas por su seguimiento.

Palabras-clave: comportamiento gobernado por las reglas, control de instrucción, comportamiento verbal, confiabilidad.

* Este projeto de pesquisa foi aprovado pelo Comitê de Ética em Pesquisa com Seres Humanos [CEP], do Centro Universitário Filadélfia [UniFil], sob o n CAAE 2719.0.000.136-10. 
Skinner $(1953 / 1989,1969)$ define regra como um estímulo discriminativo verbal que especifica uma determinada contingência. Segundo Albuquerque (2001), essa definição considera que instruções, avisos, orientações, conselhos, leis, entre outros, sejam classificados como regras, pois especificam a relação entre estímulos discriminativos, respostas e possíveis consequências de uma determinada contingência, como, por exemplo: na presença do sinal vermelho no semáforo (estímulo discriminativo), você deve parar o carro (resposta) para não bater em outro veículo (consequência). Albuquerque (2001) acrescenta que algumas regras não precisam descrever, necessariamente, toda a relação presente na contingência, especificando somente a resposta que deve ser emitida (e.g., "Silêncio") e outras descrevem a resposta e a sua provável consequência (e.g., "Andar faz bem para a saúde").

De acordo com Skinner $(1969,1974)$, regras são seguidas porque o comportamento de seguir regras foi reforçado no passado. O comportamento do ouvinte foi modelado por meio de contingências sociais a responder de acordo com as regras emitidas pelo falante, como quando uma regra é enunciada como uma ordem e as consequências aversivas para o não seguimento são organizadas pelo falante que a apresentou. Esta definição torna a regra um operante de ordem superior e as consequências sociais organizadas para o seguimento como as mantenedoras deste. Portanto, regras são seguidas porque o comportamento de seguir regras foi positivamente reforçado no passado. Nessa perspectiva, autores como Braam e Malott (1990), Mistr e Glenn (1992) têm sugerido que a história de reforço social vivida pelo indivíduo determina tal comportamento. Conforme afirma Albuquerque (2001, p. 133), "as consequências atuais especificadas na regra têm pouco efeito sobre o comportamento sob controle antecedente de regras", podendo alterar a probabilidade futura de alguns comportamentos, mas não no presente, já que essa seria determinada pela história do ouvinte.

De acordo com Abreu-Rodrigues e SanabioHeck (2004), por volta da década de 1980, alguns autores ${ }^{1}$ desenvolveram estudos com o objetivo de

1 Ayllon e Azrin, 1964; Baron, Kaufman e Stauber, 1969; Buskist e Miller, 1986; Cerutti, 1991; Danforth, Chase, entender sob quais condições a manutenção do seguimento de regra é mais (ou menos) provável, passo vital, segundo Paracampo e Albuquerque (2004), para a compreensão do comportamento governado por regras. Contudo, Schlinger e Blakely (1987), Schlinger (1993), Catania (1999) e Albuquerque (2001) apresentam definições de regras, denotando controvérsias sobre como elas funcionam.

Enquanto alguns autores (Cerutti, 1989; Galizio, 1979; Okougui, 1999) concordam com Skinner $(1980,1982)$ que regras funcionam como estímulos discriminativos, outros (Blakely \& Schlinger, 1987; Schlinger \& Blakely, 1987) sugerem que regras funcionam como estímulos alteradores de função e não como estímulos discriminativos, e há ainda outros que argumentam que regras podem funcionar como operações estabelecedoras (Hayes, Zettle \& Rosenfarb, 1989; Malott, 1989). (Albuquerque, 2001, p. 132)

Ao apresentar o comportamento verbal como um comportamento operante modelado e mantido pelas consequências mediadas por um ouvinte pertencente à mesma comunidade verbal do falante, Skinner (1957) aponta que a confiabilidade é uma variável significativa para o seguimento de regra e que, dependendo da correspondência entre a resposta verbal e a consequência especificada, o falante passa a ser confiável ou não ao ouvinte.

Além dos fatores usuais que afetam o comportamento do ouvinte (tal como a clareza do estímulo verbal ou a extensão do condicionamento de respostas separadas), uma instrução bem-sucedida está sujeita a várias condições. Uma delas é o "prestígio" do falante ou a "crença" do ouvinte acerca do que o falante diz. (Skinner, 1957, p. 436)

Dolan e Joyce, 1990; DeGrandpre e Buskist, 1991; Dixon e Hayes, 1998; Galizio, 1979; Hackenberg e Joker, 1994; Hayes, Brownstein, Zettle, Rosenfarb e Korn, 1986; Kaufman, Baron e Kopp, 1966; LeFrancois, Chase e Joyce, 1988; Lippman e Meyer, 1967; Madden, Chase e Joyce, 1998; Martinez-Sanchez e Ribes-Iñesta, 1996; Matthews, Shimoff, Catania e Sagvolden, 1977; Newman, Buffington e Hemmes, 1995; Newman, Hemmes, Buffington e Andreopoulos, 1994; Otto, Torgrud e Holborn, 1999; Raia, Shillingford, Miller e Baier, 2000; Schmitt, 1998; Shimoff, Catania e Matthews, 1981; Skinner, 1969; Wulfert, Greenway, Farkas, Hayes e Dougher, 1994. 
Sobre o seguimento de regra, estudos históricos da área (Baron, Kaufman \& Stauber, 1969; Galizio, 1979; Hayes, Brownstein, Zettle, Rosenfarb \& Korn, 1986; Joyce \& Chase, 1990; LeFrancois, Chase \& Joyce, 1988; Shimoff, Catania \& Matthews, 1981; Weiner, 1970, citados por Paracampo \& Albuquerque, 2004, p. 236) apontam que o "comportamento estabelecido por regra frequentemente não muda acompanhando mudanças nas contingências de reforço programadas em situações experimentais", a não ser que este mantenha contato com as consequências que contradigam a regra, podendo mudar em função das contingências.

Alguns desses estudos (Baron et al., 1969; Galizio, 1979; Weiner, 1970) também fazem considerações sobre o controle instrucional e apontam que as instruções consideradas correspondentes dizem respeito àquelas que especificam as reais contingências em efeito, enquanto as discrepantes, conforme afirmam Baron e Galizio (1983), são aquelas que não correspondem às reais contingências em efeito. Baron et al. (1969), Galizio (1979) e Weiner (1970) apontam que instruções correspondentes tendem a estabelecer a forma inicial do comportamento, permitindo que este fique sob controle das contingências programadas, enquanto que Baron e Galizio (1983) afirmam que instruções discrepantes podem interferir com o controle exercido pelas contingências de reforço, favorecendo um desempenho mais parecido com as contingências em vigor, sugerindo maior variabilidade comportamental.

Paracampo e Albuquerque (2004, p. 41) complementam, mais especificamente, que o seguimento de instruções é mantido a depender das contingências sociais envolvidas, das "consequências produzidas pelo seguimento" e da "magnitude das consequências por ele produzidas". (Albuquerque, Paracampo \& Albuquerque, 2004).

Diante da dificuldade em delimitar as variáveis de controle do comportamento de seguir regras, já que estas podem exercer múltiplas funções, como as descritas anteriormente, Paracampo e Albuquerque (2004) sugerem delineamento experimental no qual um mesmo sujeito fosse exposto a consequências programadas para o comportamento de seguir regras, mas também às consequências programadas para o comportamento de não seguir regras.
Este artigo, portanto, teve como objetivo analisar os efeitos de instruções correspondentes/discrepantes sobre o comportamento de seguir regras em condições com reforço imediato (reforçamento diferencial) e na ausência de reforçamento diferencial. Para tanto, foram conduzidos dois experimentos nos quais foi manipulada gradativamente a porcentagem de correspondência entre a instrução e a consequência para o comportamento de seguir regras, no Experimento I, e de seguir e não seguir, no Experimento II.

\section{Experimento I}

\section{Participantes}

Oito crianças em idade escolar (cinco a nove anos), sendo seis do sexo masculino e duas do sexo feminino, alunos de uma escola particular. Os participantes foram separados em dois grupos (Grupo $1 \mathrm{e}$ Grupo 2), com quatro crianças cada.

\section{Situação Experimental}

O participante entrava na sala, era recepcionado por dois experimentadores, escutava a instrução inicial dada pelo fantoche (falante) através de um vídeo apresentado na tela e, após cada regra enunciada, deveria escolher um quadrado dentre os dois disponíveis.

Os participantes foram individualmente alocados em uma sala de aula, sentados em frente à tela de um notebook. O comportamento do participante consistia em tocar com a mão sobre uma das cores que aparecia na tela do notebook. Ele executava uma atividade de escolha, na qual deveria escolher um dentre dois quadrados coloridos (azul e vermelho).

Dois pesquisadores permaneceram na sala durante todo o experimento, sendo que um permaneceu ao lado do participante para manusear o teclado e apresentar o brinde e outro preencheu uma folha de registro com os comportamentos emitidos pelo participante. As sessões tiveram duração total de aproximadamente 15 a 20 minutos, tendo sido filmadas por um dos experimentadores e executadas ininterruptamente ao longo de um mesmo dia.

Os demais participantes dos grupos permaneciam em um espaço da escola destinado a brincadeiras no horário de almoço, sendo chamados individualmente para a tarefa. 


\section{Consequências programadas para o seguimento de regra}

Os brindes escolhidos, com base na faixa etária das crianças, foram caixas de lápis de cor e giz de cera, juntamente com adesivos e desenhos para colorir. As caixas foram dadas a todos os participantes no término do experimento pela participação e os adesivos e desenhos eram disponibilizados de acordo com a contingência programada pelos experimentadores.

\section{Procedimento}

Para ambos os grupos ( 1 e 2), o experimento foi constituído por três fases. Os participantes do Grupo 1 (P1, P2, P3 e P4) foram submetidos à sequência de fases A-B-C e os participantes do Grupo 2 (P5, P6, P7 e P8) foram submetidos à sequência C-B-A, cada fase composta por duas condições: condição de Treino e condição de Teste, sendo que ambas as condições envolviam a exposição do participante a 10 regras (atividades de escolha).

\section{Descrição da apresentação de uma regra}

Entende-se por regra ${ }^{2}$ a seguinte sequência de eventos: a apresentação da regra propriamente dita pelo fantoche ("Escolha o quadrado azul (vermelho) que você ganha"), a resposta de escolha do participante e as consequências programadas para o seguimento, reforço diferencial (apresentação do brinde), e para o não seguimento, apresentação imediata da próxima regra (sem reforçamento diferencial).

As cores enunciadas na regra, bem como a ordem de disposição dos quadrados em cada slide (esquerda e direita) foram elencadas de forma randômica durante a elaboração do procedimento. A seguir, serão descritas as fases a que os participantes foram expostos.

2 No presente artigo, o termo regra foi utilizado para se referir à apresentação breve e direta da descrição de contingência, feita ao longo de ambos os procedimentos, antes da atividade de escolha propriamente dita. O termo instrução referiu-se à descrição mais extensa feita pelo fantoche antes do início das condições de Treino e de Teste em ambos os procedimentos. Tais termos são entendidos como sinônimos, a partir da literatura, porém foram nomeados dessa forma para fins didáticos.
Fase A

A condição de Treino era precedida pela instrução dada pelo fantoche: "Aqui na tela vão aparecer duas cores: vermelho e azul. Você tem que escolher uma cor e colocar a mão em cima dela. Se você escolher a cor premiada, você ganha um brinde na hora. Eu vou te ajudar a escolher as cores. Você pode seguir ou não os meus conselhos, vamos começar?". Nessa condição, todas as respostas do participante condizentes com a regra exposta pelo fantoche eram consequenciadas com reforço imediato (reforçamento diferencial).

A condição de Teste era precedida pela instrução: "Agora a brincadeira muda. Se você acertar a cor premiada, ganha o brinde daqui uma semana. Então, se você escolher a cor e ela for a premiada, daqui uma semana você recebe o brinde. Ninguém vai avisar se você acertou ou não. Eu vou te ajudar a escolher as cores. Você pode seguir ou não os meus conselhos, vamos começar?". O reforçamento diferencial para as respostas de escolha emitidas pelo participante era suspenso (0\%) nessa condição.

\section{Fase B}

Foi mantido o procedimento da Fase A, exceto que apenas $50 \%$ das regras emitidas pelo fantoche estavam programadas para receber reforço imediato. Foram consequenciadas as respostas condizentes com a primeira, a terceira, a quinta, a oitava e a nona regra. A escolha dessas regras foi feita através de um sorteio. As outras respostas do participante, independente de serem condizentes ou não, não recebiam reforçamento diferencial.

\section{Fase C}

Nesta condição, nenhuma resposta condizente ou não foi reforçada (manteve-se o nome da condição de Treino para facilitar a exposição dos dados, embora a ausência de reforçamento diferencial caracterizasse Teste e não Treino). $\mathrm{O}$ procedimento na condição de Teste foi mantido o mesmo das outras fases. 


\section{Resultados}

No Experimento I, cada participante poderia apresentar um máximo de 60 respostas de seguimento de regra ao longo do experimento, somando assim 240 respostas para cada grupo, 120 respostas na condição de Treino e 120 respostas na condição de Teste.

A Tabela 1 apresenta o total de seguimentos de regras do Grupo 1 (individual/grupo).

\section{Tabela 1 - Total de Seguimentos de Regras do Grupo 1 do Experimento I}

\begin{tabular}{llllllll}
\hline $\begin{array}{l}\text { Partici- } \\
\text { pantes }\end{array}$ & Fase A & \multicolumn{2}{c}{ Fase B } & \multicolumn{2}{c}{ Fase C } & Total \\
\hline & Treino & Teste & Treino & Teste & Treino & Teste \\
P1 & 10 & 10 & 9 & 10 & 10 & 9 & 58 \\
P2 & 10 & 10 & 10 & 10 & 10 & 10 & 60 \\
P3 & 10 & 10 & 10 & 10 & 10 & 10 & 60 \\
P4 & 10 & 9 & 9 & 10 & 10 & 10 & 58 \\
\hline Total & $\mathbf{7 9}$ & & $\mathbf{7 8}$ & & $\mathbf{7 9}$ & & $\mathbf{2 3 6}$
\end{tabular}

Do total de respostas de seguimento de regra deste grupo, 59 obtiveram consequência imediata e 177 foram expostas à ausência de reforçamento diferencial.

P1, na Fase A, seguiu 10 regras, tanto na condição de Treino quanto na condição de Teste. Na Fase $\mathrm{B}$, seguiu nove regras, recebendo quatro consequências imediatas durante o seguimento de regra; na condição de Teste, seguiu 10 regras. Na Fase C, seguiu 10 regras, em ambas as condições de ausência de reforçamento diferencial.

P2, na Fase A, teve desempenho semelhante ao P1 nas condições de Treino e de Teste. Na Fase B, seguiu 10 regras, recebendo cinco consequências imediatas durante o seguimento; seguiu 10 regras ainda na condição de Teste dessa fase e daquelas da Fase C. P3 teve desempenho idêntico a P2 ao longo das fases e nas respectivas condições de Treino e Teste.

P4, na Fase A, seguiu 10 regras, sendo consequenciado em todas elas e, na condição de Teste, seguiu nove regras. Na Fase B, seguiu nove regras, recebendo cinco consequências imediatas durante o seguimento; na condição de Teste, seguiu 10 regras. Na Fase $C$, teve desempenho idêntico ao apresentado por P2 e P3 nas condições de Treino e Teste dessa fase.

A Tabela 2 apresenta o total de seguimentos de regras do Grupo 2 (individual/grupo).
Tabela 2 - Total de Seguimentos de Regras Grupo 2 do Experimento I

\begin{tabular}{llllllll}
\hline $\begin{array}{l}\text { Partici- } \\
\text { pantes }\end{array}$ & Fase C & \multicolumn{2}{l}{ Fase B } & \multicolumn{2}{l}{ Fase A } & Total \\
\hline & Treino & Teste & Treino & Teste & Treino & Teste \\
P5 & 10 & 9 & 9 & 10 & 10 & 10 & 58 \\
P6 & 7 & 9 & 10 & 9 & 8 & 7 & 50 \\
P7 & 9 & 9 & 10 & 10 & 10 & 10 & 58 \\
P8 & 10 & 10 & 10 & 10 & 10 & 10 & 60 \\
\hline Total & 73 & & 78 & & 75 & & 226
\end{tabular}

Do total de respostas de seguimento de regra deste grupo, 58 obtiveram consequência imediata e 168 foram expostas à ausência de reforçamento diferencial.

P5, na Fase C, seguiu 10 regras na condição de Treino e, na condição de Teste, seguiu nove regras. $\mathrm{Na}$ Fase B, seguiu nove regras, recebendo cinco consequências imediatas durante o seguimento; na condição de Teste, seguiu 10 regras, bem como nas condições de Treino e Teste da Fase A.

P6, na Fase C, seguiu sete regras na condição de Treino e, na condição de Teste, seguiu nove regras. Na Fase B, seguiu 10 regras, recebendo cinco consequências imediatas durante o seguimento; na condição de Teste, seguiu nove regras. Na Fase A, seguiu oito regras, todas com consequências imediatas e, na condição de Teste, seguiu sete regras.

P7, na Fase C, seguiu nove regras, tanto na condição de Treino quanto na de Teste. Na Fase B, seguiu 10 regras, recebendo cinco consequências imediatas durante o seguimento; na condição de Teste, seguiu 10 regras, bem como nas condições de Treino e Teste da Fase A. P8, na Fase C, seguiu 10 regras, tanto na condição de Treino quanto na de Teste. Na Fase B e C, esse participante teve desempenho idêntico a P7 nas condições de Treino e Teste.

\section{Discussão}

Os resultados apresentados nas Tabelas 1 e 2 mostram que as diferenças de desempenho encontradas entre o Grupo 1 e o Grupo 2 foram mínimas, o que remete à hipótese de que o controle da instrução dada pelo fantoche se manteve eficiente sobre o comportamento de seguir regras dos participantes, independentemente das consequências planejadas. Essa igualdade foi encontrada tanto em condições com reforço imediato quanto na ausência de refor- 
çamento diferencial. Supõe-se que o reforçamento consistente do seguimento de regra, como comportamento a ser consequenciado, caracterizou-se como variável mantenedora.

Além disso, a análise dos dados mostra que o controle da regra foi potencializado por outras duas variáveis: a primeira refere-se às propriedades do contexto em que a regra foi apresentada, um contexto com uma história de reforçamento de seguimento de regra (dentro de uma sala de aula), o que provavelmente a potencializou. Uma segunda possível variável diz respeito à quantidade de respostas disponíveis que o participante poderia emitir quando o seguimento de regra não fosse consequenciado. Os participantes em questão mostraram que quando a resposta condizente com a regra não foi consequenciada, ocorreu uma variação e, se essa variação não fosse consequenciada diferencialmente, a ressurgência do seguimento de regra ocorreria com poucas respostas diferentes da resposta especificada pelo falante.

Nesse experimento, as contingências programadas não alteraram a probabilidade, diminuindo ou aumentando, da resposta de seguimento de regra, havendo um desempenho de altas taxas de respostas de seguimento, independente da condição experimental programada, com reforço imediato ou ausência de reforçamento diferencial. Pode-se notar que a taxa de seguimento de regra foi alta para ambos os grupos, embora o Grupo 2 tenha tido mais respostas de não seguimento do que as apresentadas pelo Grupo 1. Esse dado pode ser corroborado a partir da formulação de que, no início do experimento, o Grupo 2 foi exposto a $0 \%$ de consequenciação das respostas de seguimento de regra, variável que pode ter contribuído para a oscilação no desempenho apresentado pelo grupo nesse experimento.

A afirmação de Baron e Galizio (1983) a respeito do desempenho experimental diante de instruções discrepantes, as quais podem favorecer para que o responder seja mais parecido com as contingências em vigor, em detrimento das programadas, corrobora o dado observado no Grupo 2, tendo as crianças apresentado menores taxas de seguimento de regra e um padrão mais oscilante, comportando-se sob controle das contingências, em detrimento da regra.
A presença dos experimentadores tendo sido verbalizada no início do procedimento pode ter alterado a probabilidade de manutenção do seguimento de regra, dado este que Albuquerque, Paracampo e Albuquerque (2004) apontam como relevante, ao indicar que o seguimento tende a ser mantido quando o comportamento especificado pela instrução é monitorado por membros da comunidade verbal, tidos como "autoridades". Esses autores ainda afirmam que a monitorização exerce controle sobre as respostas apresentadas pelos participantes a depender da consequência obtida pelo seguimento de instruções, podendo não se manter quando resultar em perda de reforçadores. No Experimento I, por não envolver perdas de reforçadores, mas apenas a não apresentação destes, o seguimento se manteve.

Nesse experimento, a regra emitida pelo fantoche envolveu, provavelmente, estimulação aversiva. Esta variável pode ter sido uma condição relevante para o seguimento de regra. Analisando a função da fala do fantoche, a qual especificava a resposta e a consequência para o ouvinte, podemos afirmar que se tratava de um mando. Tendo como referência da literatura o fato de que, na instrução dada pelo falante, o mando apenas beneficia o falante, as crianças apresentaram respostas de seguimento de regra por este estar associado à consequências potencialmente aversivas. Esse seguimento seria o primeiro repertório desenvolvido pelas crianças, devido aos pais e cuidadores exercerem a função de autoridades na comunidade verbal em questão, uma vez que a população pesquisada apresentava faixa etária entre cinco e nove anos. Pesquisas que insiram breve entrevista com os participantes após o experimento podem dar mais subsídios para afirmações sobre a função do comportamento verbal.

Outra variável que pode ter contribuído para o seguimento de regra diz respeito à função, exercida pelo experimentador, de estímulo discriminativo e monitor da resposta. Segundo Faleiros e Hübner (2007), quando o experimentador participa de todas as etapas do experimento, pode adquirir função discriminativa para o seguimento de regra. Albuquerque et al. (2004) salientam que o experimentador pode adquirir, além de função discriminativa, função de monitor da resposta, a qual tem o seu controle intensificado, como nesse caso. 
Contudo, essa monitorização não potencializa o efeito da regra em situações nas quais ocorre a perda de reforço, condição ausente nesse experimento, o que se associa a outra variável relevante referente à magnitude do reforço disponibilizado. Os brindes entregues como consequência no Experimento I não eram escolhidos pelas crianças, mas arbitrariamente dispostos próximos ao notebook pelo experimentador, de acordo com cada condição. Os brindes eram semelhantes para todos os participantes, os quais não poderiam escolher entre uma variedade desses objetos, afinal estes já estavam estabelecidos. Supõe-se que tais brindes possam não ter tido uma magnitude que de fato tivesse efeito reforçador para as crianças, tendo o valor de recompensa em detrimento de reforço ao comportamento.

Pode-se hipotetizar, portanto, que a despeito das contingências programadas pelos experimentadores, o comportamento do ouvinte esteve sob controle de variáveis sociais e de consequências naturais advindas do contexto no qual os participantes estavam inseridos, no caso o ambiente escolar. Neste ambiente, que é constituído por professores e diretores (autoridades) pertencentes à comunidade verbal das crianças, o comportamento de seguir regras é fortalecido. A presença dos experimentadores supostamente favoreceu para que as crianças seguissem a regra por reforçamento negativo, uma vez que estes podem participar de uma classe de estímulos equivalentes a professores e diretores, tendo contribuído para que o ambiente se tornasse aversivo diante da monitorização. Dessa maneira, parar de se comportar era menos provável, pois elas estavam na escola e junto com pessoas supostamente punitivas.

Uma última hipótese para o padrão de resposta obtido no Experimento I diz respeito à impossibilidade de ganhar pontos se engajando em uma resposta diferente da instruída pelo fantoche, uma vez que houve ausência de reforçamento diferencial em caso de não seguimento de regra. Essa condição experimental contribuiu para a alta frequência de taxas de respostas de seguimento emitidas pelas crianças, dado discutido no Experimento II. O Experimento II teve como objetivo verificar os efeitos de instruções correspondentes/discrepantes sobre o comportamento de seguir regras em condições com reforço imediato e na ausência de refor- çamento diferencial, porém com possibilidade de consequenciação de respostas tanto de seguimento como de não seguimento de regra.

\section{Experimento II}

\section{Participantes}

Oito crianças em idade escolar (seis a nove anos), sendo uma do sexo masculino e sete do sexo feminino, tendo sido separadas de maneira idêntica à do Experimento I.

\section{Situação Experimental}

Os participantes foram individualmente alocados no Laboratório de Psicologia Humana e Experimental do Centro Universitário Filadélfia. Ao entrar no local, o participante se posicionava no centro da sala do laboratório. O comportamento deste consistia em tocar com a mão sobre uma das mesas dispostas na sala. Ele executava uma atividade de escolha, na qual deveria escolher uma dentre quatro mesas coloridas (verde, azul, amarelo, laranja). Tal atividade envolvia topografia de resposta diferente da solicitada no Experimento I, delimitando maior número de possibilidades de escolha aos participantes desse experimento.

Além das quatro mesas, havia um anteparo com um fantoche manipulado pelo experimentador, tendo sido o único pesquisador a permanecer no local durante todo o experimento. O experimentador se posicionou distante do participante, evitou contato visual e qualquer interação verbal além das regras. A consequência era depositada em um recipiente localizado ao lado do experimentador. Tais alterações foram necessárias para que sua presença não potencializasse o efeito da regra pela monitorização, como no Experimento I.

O participante entrava na sala, escutava a instrução inicial dada pelo fantoche posicionado atrás do anteparo e, após cada regra enunciada, deveria escolher uma mesa dentre as quatro disponíveis. As sessões foram filmadas e executadas ininterruptamente.

\section{Consequências programadas para o seguimento de regra}

Os brindes escolhidos foram balas e brinquedos adequados à idade das crianças conforme a classificação do Instituto Nacional de Metrologia, 
Qualidade e Tecnologia [INMETRO], sendo estes diferentes dos brindes disponibilizados no Experimento I, mas administrados de forma semelhante (pela participação e nas condições experimentais).

\section{Sequência experimental}

No Experimento II, as condições de Treino e de Teste corresponderam parcialmente às descritas no Experimento I, pois no Experimento II houve reforçamento diferencial para o não seguimento de regra, o qual obteve consequências imediatas nas condições de Treino.

\section{Procedimento}

O procedimento empregado nesse experimento foi semelhante ao utilizado no Experimento I, com duas distinções: (a) Diante da emissão de regras que não condiziam às contingências de reforço programadas, existia a oportunidade de o participante receber consequências escolhendo uma das três mesas diferentes daquela que havia sido enunciada pela regra; (b) Houve a exposição dos participantes a uma quarta fase (Fase D), que consistia na repetição da primeira fase do experimento. Para o Grupo 1 , a condição referiu-se a $100 \%$ de regras verdadeiras e para o Grupo 2, a condição foi de $0 \%$ de regras verdadeiras. Portanto, os participantes do Grupo 1 (P9, P10, P11 e P12) foram submetidos à sequência de fases A-B-C-D e os participantes do Grupo 2 (P13, P14, P15 e P16) à sequência C-B-A-D.

\section{Descrição da apresentação de uma regra}

Entende-se por regra a seguinte sequência de eventos: a apresentação da regra propriamente dita pelo fantoche ("Escolha a mesa azul [verde, amarela, laranja] que você ganha"), a resposta de escolha do participante e as consequências programadas para o seguimento e/ou não seguimento da regra emitida pelo fantoche. A escolha das cores enunciadas na regra e a disposição das mesas na sala do laboratório foram definidas de maneira idêntica à do Experimento I (randomicamente).

A condição de Treino era precedida pela instrução dada pelo fantoche: "Agora vamos fazer uma brincadeira. Quando eu falar já, você deve escolher uma das mesas e colocar a mão em cima dela. Se escolher a mesa premiada, ganhará um brinde na hora. Depois de escolher a mesa e de receber os brindes, volte para o meio da sala para começarmos de novo. Eu sou um fantoche que vai ajudar você a escolher as mesas. Você pode seguir ou não os meus conselhos, vamos começar?".

A condição de Teste era precedida pela instrução: "Agora a brincadeira muda um pouco. Quando eu falar já, você deve escolher uma das mesas e colocar a mão em cima dela. Se escolher a mesa premiada, ganhará o brinde daqui uma semana. Então, se você acertar, daqui uma semana trago os brindes. Eu não vou avisar se você acertou ou não. Eu sou um fantoche que vai ajudar você a escolher as mesas. Você pode seguir ou não os meus conselhos, vamos começar?".

\section{Resultados}

No Experimento II, cada sujeito poderia apresentar um máximo de 80 respostas de seguimento de regra ao longo do experimento, somando assim 320 respostas para cada grupo, 160 respostas na condição de Treino e 160 respostas na condição de Teste.

A Tabela 3 apresenta o total de seguimentos de regras do Grupo 1 (individual/grupo).

Do total de respostas de seguimento de regra deste grupo, 66 obtiveram consequência imediata e 86 foram expostas à ausência de reforçamento diferencial. Em relação às respostas de não seguimento, estas totalizaram 19 respostas, presentes nas fases $\mathrm{B}$ e C.

P9, na Fase A, seguiu 10 regras, tanto na condição de Treino quanto na condição de Teste. Na Fase $B$, seguiu três regras, recebendo uma consequência imediata durante o seguimento de regra e uma consequência quando do não seguimento; na condição de Teste, seguiu duas regras. Na Fase C, seguiu uma regra, recebendo duas consequências imediatas durante o não seguimento; na condição de Teste, seguiu uma regra. Na Fase D, seguiu 10 regras tanto na condição de Treino, sendo consequenciado em todas elas, quanto na condição de Teste.

P10, na Fase A, teve desempenho semelhante a P9 nas condições de Treino e de Teste. Na Fase B, seguiu duas regras, recebendo uma consequência imediata durante o não seguimento; na condição de Teste, seguiu cinco regras. Na Fase C, seguiu uma regra, recebendo seis consequências imediatas durante o não seguimento; na condição de Teste, 
Tabela 3 - Total de Seguimentos de Regras Grupo 1 do Experimento II

\begin{tabular}{llllllllll}
\hline Participantes & Fase A & & Fase B & & Fase C & Fase D & Total \\
\hline & Treino & Teste & Treino & Teste & Treino & Teste & Treino & Teste \\
P9 & 10 & 10 & 3 & 2 & 1 & 1 & 10 & 10 & 47 \\
P10 & 10 & 10 & 2 & 5 & 1 & 2 & 8 & 1 & 39 \\
P11 & 6 & 4 & 4 & 4 & 2 & 1 & 3 & 3 & 27 \\
P12 & 7 & 2 & 6 & 5 & 4 & 3 & 6 & 6 & 39 \\
\hline Total & 59 & & 31 & & 15 & & 47 & 152
\end{tabular}

seguiu duas regras. Na Fase D, seguiu oito regras, sendo consequenciado em todas elas, e na condição de Teste, seguiu uma regra.

P11, na Fase A, seguiu seis regras, sendo consequenciado em todas elas; na condição de Teste, seguiu quatro regras. Na Fase B, seguiu quatro regras, recebendo três consequências imediatas durante o seguimento e uma consequência quando do não seguimento; na condição de Teste, seguiu quatro regras. Na Fase C, seguiu duas regras, recebendo duas consequências imediatas durante o não seguimento; na condição de Teste, seguiu uma regra. $\mathrm{Na}$ Fase $\mathrm{D}$, seguiu três regras tanto na condição de Treino, sendo consequenciado em todas elas, quanto na condição de Teste.

P12, na Fase A, seguiu sete regras, sendo consequenciado em todas elas; na condição de Teste, seguiu duas regras. Na Fase B, seguiu seis regras, recebendo duas consequências imediatas durante o seguimento e duas consequências quando do não seguimento; na condição de Teste, seguiu cinco regras. Na Fase $\mathrm{C}$, seguiu quatro regras, recebendo quatro consequências imediatas durante o não seguimento; na condição de Teste, seguiu três regras. $\mathrm{Na}$ Fase $\mathrm{D}$, seguiu seis regras tanto na condição de Treino, sendo consequenciado em todas elas, quanto na condição de Teste.
A Tabela 4 apresenta o total de seguimentos de regras do Grupo 2 (individual/grupo).

Do total de respostas de seguimento de regra deste grupo, 31 obtiveram consequência imediata e 90 foram expostas à ausência de reforçamento diferencial. Em relação às respostas de não seguimento, estas totalizaram 30 respostas, presentes nas fases $\mathrm{C}, \mathrm{B}, \mathrm{A}$ e $\mathrm{D}$.

P13, na Fase C, seguiu três regras, recebendo sete consequências imediatas durante o não seguimento; na condição de Teste, seguiu três regras. Na Fase B, seguiu quatro regras, recebendo uma consequência imediata durante o seguimento e uma consequência quando do não seguimento; na condição de Teste, seguiu três regras. Na Fase A, seguiu oito regras, sendo consequenciado em todas elas, recebendo duas consequências durante o não seguimento, e na condição de Teste, seguiu três regras. Na Fase D, seguiu três regras, recebendo cinco consequências imediatas durante o não seguimento; na condição de Teste, seguiu quatro regras.

P14, na Fase C, seguiu 10 regras na condição de Treino e, na condição de Teste, seguiu nove regras. $\mathrm{Na}$ Fase B, seguiu uma regra, recebendo uma consequência imediata durante o seguimento e quatro consequências quando do não seguimento; na condição de Teste, seguiu duas regras. Na Fase A,

Tabela 4 - Total de Seguimentos de Regras Grupo 2 do Experimento II

\begin{tabular}{llllllllll}
\hline Participantes & Fase C & & Fase B & & Fase A & Fase D & Total \\
\hline & Treino & Teste & Treino & Teste & Treino & Teste & Treino & Teste \\
P13 & 3 & 3 & 4 & 3 & 8 & 3 & 3 & 4 & 31 \\
P14 & 10 & 9 & 1 & 2 & 5 & 0 & 2 & 4 & 33 \\
P15 & 3 & 1 & 3 & 1 & 5 & 0 & 4 & 3 & 20 \\
P16 & 2 & 4 & 7 & 2 & 5 & 9 & 4 & 4 & 37 \\
\hline Total & $\mathbf{3 5}$ & & $\mathbf{2 3}$ & & $\mathbf{3 5}$ & & $\mathbf{2 8}$ & $\mathbf{1 2 1}$
\end{tabular}


seguiu cinco regras, sendo consequenciado em todas elas; na condição de Teste, não seguiu nenhuma regra. Na Fase D, seguiu duas regras, recebendo três consequências imediatas durante o não seguimento; na condição de Teste, seguiu quatro regras.

P15, na Fase C, seguiu três regras na condição de Treino e, na condição de Teste, seguiu uma regra. Na Fase B, seguiu três regras, recebendo duas consequências imediatas durante o seguimento e duas consequências quando do não seguimento; na condição de Teste, seguiu uma regra. Na Fase A, seguiu cinco regras, sendo consequenciado em todas elas; na condição de Teste, não seguiu nenhuma regra. Na Fase D, seguiu quatro regras, recebendo três consequências imediatas durante o não seguimento; na condição de Teste, seguiu três regras.

P16, na Fase C, seguiu duas regras, recebendo duas consequências imediatas durante o não seguimento; na condição de Teste, seguiu quatro regras. $\mathrm{Na}$ Fase B, seguiu sete regras, recebendo quatro consequências imediatas durante o seguimento e uma consequência quando do não seguimento; na condição de Teste, seguiu duas regras. Na Fase A, seguiu cinco regras, sendo consequenciado em todas elas; na condição de Teste, seguiu nove regras. $\mathrm{Na}$ Fase D, seguiu quatro regras, recebendo duas consequências imediatas durante o não seguimento; na condição de Teste, seguiu quatro regras.

A Tabela 5 apresenta o total de seguimentos de regras nas condições de Treino e Teste.

\section{Discussão}

Tabela 5 - Total de Seguimentos de Regras dos Participantes nas Condições de Treino e Teste

\begin{tabular}{llll}
\hline Experimento & Participantes & Condições \\
\hline \multirow{3}{*}{ Exp. I } & Grupo 1 & Treino & Teste \\
& Grupo 2 & 118 & 118 \\
Exp. II & Grupo 1 & 83 & 113 \\
& Grupo 2 & 69 & 69 \\
\hline & Total & 383 & 352 \\
\hline
\end{tabular}

Pode-se verificar que, conforme hipotetizado, o Experimento I promoveu condições de ocorrência de maior taxa de respostas de seguimento de regra do que o obtido no Experimento II, sendo que, nes- te último, estas favoreceram a variabilidade comportamental.

Os resultados dos participantes do Grupo 1 do Experimento II, em comparação aos resultados dos participantes do Grupo 2, sugerem que o efeito da consequência imediata foi uma variável importante para que as taxas de seguimentos de regras, para ambos os grupos, fossem mais altas nessa condição, em comparação àquelas apresentadas na ausência de reforçamento diferencial. Com exceção dos participantes P10 e P16, que apresentaram maiores taxas de seguimentos de regras na ausência de reforçamento diferencial, e P11 que manteve sua taxa de emissão de seguimento estável nas duas condições, os demais apresentaram maiores taxas de seguimento diante de consequências imediatas.

Com relação aos desempenhos dos dois grupos em todas as fases nas condições experimentais (reforço imediato e ausência de reforçamento diferencial), os participantes do Grupo 1 apresentaram maiores taxas de seguimentos de regras em relação às emitidas pelo Grupo 2, tanto diante de consequências imediatas quanto na ausência de reforçamento diferencial, o que sugere que as contingências programadas com relação à confiabilidade no falante (as sequências de regras verdadeiras ou falsas e a consequenciação do não seguimento) determinaram tais padrões. Nesse caso, portanto, a sequência experimental estaria vinculada à relação de confiabilidade construída entre falante e ouvinte.

A hipótese sobre a relação de confiabilidade pode ser elaborada a partir dos dados referentes às taxas de seguimento apresentadas pelos participantes do Grupo 1, os quais iniciaram o experimento obtendo $100 \%$ de consequenciação para o seguimento de regra (Fase $\mathrm{A}$ ), em relação às emitidas pelos participantes do Grupo 2, que obtiveram 0\% (Fase C). Os participantes do Grupo 1 apresentaram maiores taxas de seguimento do que as do Grupo 2.

Nesse aspecto, o que pode se observar é que essa relação de confiabilidade que foi construída nos primeiros contatos do ouvinte com as regras enunciadas pelo falante pode ser alterada, a depender das contingências apresentadas a este, podendo ocorrer o esvanecimento desta relação. No Grupo 1, quando submetidos à Fase B, os participantes P9, P10 e P11 apresentaram uma queda no seguimento 
de regra. Isto implica dizer que, quando a confiabilidade no falante foi reduzida para $50 \%$, a adesão dos ouvintes diminuiu em relação à Fase A. Esse dado sugere que, de fato, a consequência teve efeito (com valor reforçador) e a história de reforçamento da fase anterior também se caracterizou como variável relevante.

Os participantes P14 e P15 não apresentaram aumento na taxa de seguimento de regra com a mudança de $0 \%$ para $50 \%$ na probabilidade de consequenciação do mesmo. O efeito da Fase C, disposta no início do experimento, com $0 \%$ de probabilidade de obtenção de consequência para a resposta de seguir a regra mostra que, quanto mais regras falsas o falante emitir, mais difícil será restabelecer as taxas de seguimentos de regras (confiabilidade entre falante e ouvinte). Essa mesma fase, quando executada no Grupo 1, corrobora a afirmação de que instruções discrepantes podem promover um esvanecimento da confiabilidade construída no passado, já que todos os participantes apresentaram um alto índice de não seguimento.

Apenas dois participantes (P12 e P14) apresentaram desempenhos contrários às contingências programadas, respeitando-se as condições experimentais a que foram expostos, respectivamente, no Grupo 1 e 2, as quais envolvem outras variáveis já mencionadas. O participante $\mathrm{P} 12$, mesmo recebendo consequências imediatas para a resposta de seguir a regra, apresentou menor taxa de seguimento de regra na condição de ausência de reforçamento diferencial. O participante $\mathrm{P} 14$, mesmo não recebendo consequências imediatas para a resposta de seguir regras, apresentou maior taxa de seguimento de regra na ausência de reforçamento diferencial. Ambos os participantes, mesmo recebendo consequências pelas respostas de seguir a regra, não apresentaram um aumento no seguimento na fase seguinte.

Percebeu-se que, nesse experimento, o controle da regra foi estabelecido pela apresentação da consequência imediata. Quando a mesma era apresentada condizente ao comportamento de seguir a regra, o seguimento se manteve mesmo na ausência de reforçamento diferencial, o que não ocorreu quando as consequências foram liberadas contingentemente ao comportamento de não seguimento (perda do valor reforçador).

Portanto, verificou-se que o efeito da conse- quência imediata é fundamental no estabelecimento do comportamento de seguir regras quando a contingência envolve ausência de reforçamento diferencial e, mesmo depois de estabelecida a relação de confiabilidade entre falante (fantoche) e ouvinte (criança), tal consequência se faz necessária para a manutenção dessa relação, corroborando a afirmação de que a história de consequenciação imediata entre o falante e o ouvinte influencia no controle da regra (Skinner, 1957).

Outras variáveis manipuladas nesse segundo procedimento referem-se a menor expressividade do experimentador junto aos participantes e a oportunidade destes emitirem outra resposta (diferente da especificada pelo fantoche) quando a regra fosse emitida. Ao contrário do ocorrido no Experimento I, a interação entre experimentador e participante foi mais restrita, o que inviabilizou que o monitor da tarefa adquirisse função discriminativa para o seguimento de regra, variável importante na emissão de respostas, conforme apontado por Faleiros e Hübner (2007). Essa "ausência" favoreceu as oscilações nas taxas de seguimento.

Além disso, como estes participantes tiveram maior número de possibilidades de respostas a serem emitidas (quatro cores na mesa, em detrimento de apenas duas cores no computador), puderam variar o desempenho, sem que isso envolvesse perda de reforçadores.

Em relação aos reforçadores disponibilizados no Experimento II, além de serem brinquedos, que já se caracterizam como reforçadores condicionados utilizados na comunidade verbal das crianças da faixa etária empregada nesse estudo, estavam vinculados a reforçadores primários (comida), o que pode ter favorecido para que de fato influenciassem nos desempenhos, com valor reforçador, em detrimento dos brindes dados no Experimento I.

\section{Conclusões e Sugestões}

No estudo em questão, o comportamento do ouvinte diante do seguimento de regra foi analisado através da exposição a dois experimentos com três fases distintas para Grupo 1 e Grupo 2 no Experimento I e quatro fases distintas para outros dois grupos no Experimento II. Estas fases estabeleciam diferentes contingências de reforçamento programadas, com 
condições de reforço imediato (Treino) e ausência de reforçamento diferencial (Teste).

Tendo em vista que o objetivo desta pesquisa consistia em compreender os efeitos de instruções correspondentes/discrepantes sobre o comportamento de seguir regras em condições com reforço imediato (diferencial) e na ausência deste, elaboraram-se procedimentos envolvendo consequências diferenciais, que variavam de 0 a $100 \%$ de reforço, para o comportamento de seguir as regras. A hipótese inicial estava relacionada ao fato de que os participantes (ouvintes) tenderiam a apresentar desempenhos diferenciados, a depender das contingências programadas e do controle instrucional advindo da fala do fantoche (falante), o qual descrevia instruções e regras no início de cada condição e fase.

Dessa maneira, os participantes foram expostos a contingências programadas distintas que correspondiam $(100 \%)$ ou não $(50 \%$ e $0 \%)$ às instruções dadas pelo fantoche, considerando que estariam também expostos a reforço imediato (Treino) e ausência de reforçamento diferencial (Teste). Estas contingências poderiam alterar ou não o comportamento de seguir regras, sugerindo variáveis sobre as relações de confiabilidade.

Os participantes do Grupo 1, do Experimento II, e dos Grupos 1 e 2, do Experimento I, apresentaram uma alta porcentagem de seguimento de regra, mesmo quando a taxa de reforço imediato era menor que 100\%.Já o Grupo 2, do Experimento II, apresentou um responder diferenciado em função da frequência de consequências imediatas despendidas à resposta de seguir regras na primeira fase a que foram expostos (Fase C), na qual não houve reforçamento diferencial para o seguimento e obtiveram-se consequências para o não seguimento.

Pode-se observar que os grupos que iniciaram com a Fase A apresentaram maior consistência no seguimento de regra $(69,29 \%)$ comparados com os grupos que iniciaram com a Fase C $(61,96 \%)$. Esses resultados mostram que a confiabilidade no falante está relacionada com o fato da regra ser correspondente às consequências produzidas pelo seu seguimento.

Outras variáveis, contudo, podem ter favorecido para que se obtivessem tais desempenhos nas relações de confiabilidade, tais como: a presença ou não do experimentador, o número de respostas possíveis a serem dadas na ocasião da emissão da regra pelo fantoche (duas opções no Experimento I e quatro no II) e a condição de reforço diferencial para o não seguimento.

Diante disto, faz-se necessário, para futuras pesquisas, o delineamento de arranjo experimental que manipule com mais precisão a consequência diferencial tanto para o comportamento de seguir quanto para o comportamento de não seguir a regra, devendo estar clara a correspondência e a não correspondência entre resposta verbal e consequência.

A manipulação da monitorização da tarefa experimental em locais mais isentos de histórias pré-experimentais dos participantes, da quantidade de opções de respostas cedidas ao ouvinte e de registros sobre repertórios verbais pode complementar os dados obtidos sobre as relações de confiabilidade nesse estudo. Sugere-se, ainda, análise acerca da primeira resposta (antes da consequenciação) emitida por cada um dos participantes. A coleta minuciosa desses dados pode indicar o quanto os participantes se comportaram sob controle da regra antes mesmo da obtenção da consequência programada e de história experimental.

Estabelecer uma relação de confiabilidade é fundamental para que o ouvinte responda conforme a regra na ausência de reforçamento diferencial. Professores e demais autoridades na comunidade verbal que estabeleçam relações com base nas variáveis investigadas neste estudo podem tornar mais provável que seu aluno/colaborador responda a regras fora e dentro do contexto em questão. As mudanças nas variáveis independentes realizadas no Experimento II e o efeito obtido nos resultados sugerem que o estudo do comportamento governado verbalmente é sensível a muitas outras variáveis além do reforçamento imediato.

A presente pesquisa buscou complementar os estudos sobre correspondência entre comportamento verbal e não verbal introduzindo o conceito de confiabilidade proposto por Skinner (1957) e construindo, experimentalmente, condições que manipulassem variáveis independentes em função do comportamento de seguir regras, com situações mais aproximadas de contextos aplicados, as quais pudessem agregar, comparativamente, dados de participantes expostos à consequenciação de seguimento e de não seguimento de regra. 
Acredita-se que a ampliação dos conhecimentos extraídos de pesquisas envolvendo seguimento de regra e comportamento verbal possa vir a ser útil para outros pesquisadores que se interessem por esses assuntos, bem como pode auxiliar profissionais que atuam nos contextos clínico, organizacional e educacional a melhor entender o comportamento de seguir regras e como proporcionar contingências para a construção de relações de confiabilidade.

\section{Referências}

Abreu-Rodrigues, J. \& Sanabio-Heck, E. T. (2004). Instruções e autoinstruções: Contribuições da pesquisa básica. Em C. N. de Abreu \& H. J. Guilhardi (Orgs.). Terapia comportamental e cognitivo-comportamental: Práticas clínicas (pp. 152-168). São Paulo: Roca.

Albuquerque, L. C. (2001). Definições de regras. Em H. J. Guilhardi, M. B. B. P. Madi, P. P. Queiroz \& M. C. Scoz (Orgs.). Sobre comportamento e cognição: Expondo a variabilidade (pp. 132-140). Santo André, SP: ARBytes.

Albuquerque, N. M. A., Paracampo, C. C. P. \& Albuquerque, L. C. (2004). Análise do papel de variáveis sociais e de consequências programadas no seguimento de instruções. Psicologia: Reflexão e Crítica, 17, 31-42.

Ayllon, T. \& Azrin, N. H. (1964). Reinforcement and instructions with mental patients. Journal of the Experimental Analysis of Behavior, 7, 327-331.

Baron, A. \& Galizio, M. (1983). Instructional control of human operant behavior. The Psychological Record, 33, 495-520.

Baron, A., Kaufman, A. \& Stauber, K. A. (1969). Effects of instructions and reinforcement-feedback on human operant behavior maintained by fixed-interval reinforcement. Journal of the Experimental Analysis of Behavior, 12, 701-712.

Blakely, E. \& Schlinger, H. (1987). Rules: Functionaltering contingency-specifying stimuli. The Behavior Analyst, 10, 183-187.

Braam, C. \& Malott, R. W. (1990). "I'll do it when the snow melts": The effects of deadlines and delayed outcomes on rule-governed behavior in preschool children. The Analysis of Verbal Behavior, 8, 67-76.

Buskist, W. F. \& Miller Jr., H. L. (1986). Interaction between rules and contingencies in the con- trol of human fixed-interval performance. The Psychological Record, 36, 109-116.

Catania, A. C. (1999). Aprendizagem: Comportamento, linguagem e cognição (D. G. Souza et al., Trads.). Porto Alegre: Artmed. (Trabalho original publicado em 1998)

Cerutti, D. T. (1989). Discrimination theory of rule-governed behavior. Journal of the Experimental Analysis of Behavior, 51, 259-276.

Cerutti, D. T. (1991). Discriminative versus reinforcing properties of schedules as determinants of schedule insensitivity in humans. The Psychological Record, 41, 51-67.

Danforth, J. S., Chase, P. N., Dolan, M. \& Joyce, J. H. (1990). The establishment of stimulus control by instructions and by differential reinforcement. Journal of the Experimental Analysis of Behavior, 54, 97-112.

DeGrandpre, R. J. \& Buskist, W. F. (1991). Effects of accuracy of instructions on human behavior: Correspondence with reinforcement contingencies matters. The Psychological Record, 41, 371-384.

Dixon, M. R. \& Hayes, L. J. (1998). Effects of differing instructional histories on the resurgence of rule-following. The Psychological Record, 48, 275-292.

Faleiros, T. C. \& Hübner, M. M. C. (2007). Efeito do reforçamento diferencial de resposta verbal referente à leitura sobre a duração da resposta de ler. Revista Brasileira de Terapia Comportamental e Cognitiva, 9, 307-316.

Galizio, M. (1979). Contingency-shaped and rule-governed behavior: Instructional control of human loss avoidance. Journal of the Experimental Analysis of Behavior, 31, 53-70.

Hackenberg, T. D. \& Joker, V. R. (1994). Instructional versus schedule control of humans' choices in situations of diminishing returns. Journal of the Experimental Analysis of Behavior, 62, 367-383.

Hayes, S. C., Brownstein, A. J., Zettle R. D., Rosenfarb, I. \& Korn, Z. (1986). Rule-governed behavior and sensitivity to changing consequences of responding. Journal of the Experimental Analysis of Behavior, 45, 237-256. 
Hayes, S. C., Zettle R. D. \& Rosenfarb, I. (1989). Rule-following. Em S. C. Hayes (Org.). Rulegoverned behavior: Cognition, contingencies, and instructional control (pp. 191-220). New York: Plenum.

Joyce, J. H. \& Chase, P. N. (1990). Effects of response variability on the sensivity of rule-governed behavior. Journal of the Experimental Analysis of Behavior, 54, 251-262.

Kaufman, A., Baron, A. \& Kopp, R. E. (1966). Some effects of instructions on human operant behavior. Psychonomic Monograph Supplements, 1, 243-250.

LeFrancois, J. R., Chase P. N. \& Joyce, J. H. (1988). The effects of a variety of instructions on human fixed-interval performance. Journal of the Experimental Analysis of Behavior, 49, 383-393.

Lippman, L. G. \& Meyer, M. E. (1967). Fixedinterval performance as related to instructions and to subjects' verbalizations of the contingency. Psychonomic Science, 8, 135-136.

Madden, G. J., Chase, P. N. \& Joyce, J. H. (1998). Making sense of sensitivity in the human operant literature. The Behavior Analyst, 21, 1-12.

Malott, R. W. (1989). The achievement of evasive goals: Control by rules describing contingencies that are not direct-acting. Em S. C. Hayes (Org.). Rule-governed behavior: Cognition, contingencies, and instructional control (pp. 269322). New York: Plenum.

Martinez-Sanchez, H. \& Ribes-Iñesta, E. (1996). Interactions of contingencies and instructional history on conditional discrimination. The Psychological Record, 46, 301-318.

Matthews, B. A., Shimoff, E., Catania, A. C. \& Sagvolden, T. (1977). Uninstructed human responding: Sensitivity to ratio and interval contingencies. Journal of the Experimental Analysis of Behavior, 27, 453-467.

Mistr, K, N. \& Glenn, S, S. (1992). Evocative and function-altering effects of contingency-specifying stimuli. The Analysis of Verbal Behavior, 10, 11-21.

Newman, B., Buffington, D. M. \& Hemmes, N. S. (1995). The effects of schedules of reinforcement on instructions following. The Psychological Record, 45, 463-476.

Newman, B., Hemmes, N. S., Buffington, D. M. \& Andreopoulos, S. (1994). The effects of schedu- les of reinforcement on instruction-following in human subjects with verbal and nonverbal stimuli. The Analysis of Verbal Behavior, 12, 31-41.

Okougui, H. (1999). Instructions as discriminative stimuli. Journal of the Experimental Analysis of Behavior, 72, 205-214.

Otto, T. L., Torgrud, L. J. \& Holborn, S. W. (1999). An operant blocking interpretation of instructed insensitivity to schedule contingencies. The Psychological Record, 49, 663-684.

Paracampo, C. C. P. \& Albuquerque, L. C. de. (2004). Análise do papel das consequências programadas no seguimento de regras. Interação em Psicologia, 8, 237-245.

Raia, C. P., Shillingford, S. W., Miller Jr., H. L. \& Baier, P. S. (2000). Interaction of procedural factors in human performance on yoked schedules. Journal of the Experimental Analysis of Behavior, 74, 265-281.

Schlinger, H. D., Jr. (1993). Separating discriminative and function-altering effects of verbal stimuli. The Behavior Analyst, 16, 9-23.

Schlinger, H., \& Blakely, E. (1987). Functionaltering effects of contingency-specifying stimuli. The Behavior Analyst, 10, 41-45.

Schmitt, D. (1998). Effects of consequences of advice on patterns of rule control and rule choice. Journal of the Experimental Analysis of Behavior, 70, 1-21.

Shimoff, E., Catania, A. C. \& Matthews, B. A. (1981). Uninstructed human responding: Sensitivity of low-rate performance to schedule contingencies. Journal of the Experimental Analysis of Behavior, 36, 207-220.

Skinner, B. F. (1957). Verbal behavior. New York: Appleton-Century-Crofts.

Skinner, B. F. (1969). Contingencies of reinforcement: A theoretical analysis. New York: AppletonCentury-Crofts.

Skinner, B. F. (1974). About behaviorism. New York: Knopf.

Skinner, B. F. (1980). Contingências de reforço: Uma análise teórica (R. Moreno, Trad.). Os Pensadores. São Paulo: Abril Cultural. (Trabalho original publicado em 1969)

Skinner, B. F. (1982). Sobre o behaviorismo. São Paulo: Cultrix. (Trabalho original publicado em 1974) 
Skinner, B. F. (1989). Ciência e comportamento humano (J. C. Todorov \& R. Azzi, Trads.). São Paulo: Martins Fontes. (Trabalho original publicado em 1953)

Weiner, H. (1970). Instructional control of human operant responding during extinction following fixed-ratio conditioning. Journal of the Experimental Analysis of Behavior, 13, 391-394.

Wulfert, E., Greenway, D. E., Farkas, P., Hayes, S. C. \& Dougher, M. J. (1994). Correlation between self-reported rigidity and rule-governed insensitivity to operant contingencies. Journal of Applied Behavior Analysis, 27, 659-671.

\section{Informações do Artigo}

Histórico do artigo:

Submetido em: 13/02/2013

Primeira decisão editorial: 14/05/2013

Segunda decisão editorial: 05/06/2013

Aceito em: 18/06/2013 